\title{
THE OPTICAL AND INFRARED PHOTOMETRIC EVOLUTION OF THE RECENT STELLAR MERGER, V1309 SCO
}

\author{
Bruce McCollum $^{1,8}$, Seppo Laine ${ }^{2}$, Petri Väisänen ${ }^{3}$, Frederick C. Bruhweiler ${ }^{4}$, Lee Rottler ${ }^{5}$, \\ Stuart Ryder ${ }^{6}$, Glenn M. Wahlgren ${ }^{4}$, Sudhanshu Barway ${ }^{3}$, Takahiro Nagayama ${ }^{7}$, and Rajin Ramphul ${ }^{3}$ \\ ${ }^{1}$ California Institute of Technology, Pasadena, CA 91125, USA; mccollub@cua.edu \\ ${ }^{2}$ Spitzer Science Center, Caltech, MS 220-6, Pasadena, CA 91125, USA; seppo@ipac.caltech.edu \\ ${ }^{3}$ South African Astronomical Observatory/South African Large Telescope, P.O. Box 9, 7935 Observatory, Cape Town, South Africa \\ ${ }^{4}$ IACS/Department of Physics, Catholic University of America, Washington, DC 20064, USA; bruhweiler@cua.edu, wahlgren@cua.edu \\ ${ }^{5}$ Pisgah Astronomical Research Institute, Rosman, NC 28772, USA \\ ${ }^{6}$ Australian Astronomical Observatory, P.O. Box 915, North Ryde, NSW, Australia \\ ${ }^{7}$ Department of Astrophysics, Nagoya University, Furo-cho, Chikusa-ku, Nagoya 464-8602, Japan \\ Received 2013 September 27; accepted 2013 October 17; published 2013 December 2
}

\begin{abstract}
Nova Sco 2008 was recently shown to have resulted from the merger of the two stars in the contact binary V1309 Sco. This is the first stellar merger ever observed between two convective stars. We present archival data, new infrared photometry, and Hubble Space Telescope WFC3 imaging of V1309 Sco. Spitzer observations show that it had a large infrared excess in the $3.6 \mu \mathrm{m}$ to $8 \mu \mathrm{m}$ range more than a year before the merger. Standard color diagnostics of the pre-merger infrared colors place V1309 Sco in the same region where evolved stars with chemically complex mass loss are located. Since the nova outburst subsided in optical bandpasses in 2008, the merger remnant's brightness in optical bandpasses, near-IR bandpasses, and the Spitzer $3.6 \mu \mathrm{m}$ and $4.5 \mu \mathrm{m}$ channels has varied by several magnitudes and in complex ways. A temporary, strong increase in the reddening during 2010 suggests the occurrence of a dust formation event. We point out several peculiarities in the relative fluxes and time behavior of the optical and near-IR magnitudes, which could be explained if some of the photometric bandpasses in the $1-5 \mu \mathrm{m}$ range are strongly affected by emission lines.
\end{abstract}

Key words: binaries: general - novae, cataclysmic variables - stars: evolution - stars: individual (V1309 Sco)

Online-only material: color figure

\section{INTRODUCTION}

Stellar mergers are an important but poorly understood process in binary star evolution. Mergers are thought to be common enough to significantly affect the observable properties of many high-density stellar aggregates (e.g., Leonard 1989). It has been estimated that $\sim 10 \%$ of all stars will ultimately be involved in a merger (Pols 2009), including, for example, all low-mass contact binaries (Webbink 1976, 1985; Robertson \& Eggleton 1977; Tutukov \& Yungelson 1987; Mateo et al. 1990; Rasio 1995; Lohr et al. 2012). Several types of peculiar stars whose origins remain uncertain, e.g., Be stars, sdB stars, $B[\mathrm{e}]$ stars, and blue stragglers, have been suggested to form from mergers. Mergers may influence the observed properties of the Galactic center (i.e., the "missing giants" problem; Genzel et al. 1996; Buchholz et al. 2009; Dale et al. 2009). Mergers of pairs of low-mass stars may also be the source of some hot Jupiters with anomalously large diameters (Martin et al. 2011).

The 2008 nova V1309 Sco was recently shown (Tylenda et al. 2011) to have resulted from the merger of a contact binary. This is the first merger between two convective stars to ever be observed during the event. It represents a unique opportunity to learn about the merger process and about the observational appearance of a merger product. The pre-merger masses, spectral types, and orbit period have been estimated from pre-eruption photometric monitoring (Tylenda et al. 2011; Stepien 2011). This enhances the value of this object for modeling because these parameters influence many aspects of the merger process as well as the nature of the remnant object

\footnotetext{
8 Current address: IACS/Department of Physics, Catholic University of America, Washington, DC 20064, USA.
}

(e.g., Freitag \& Benz 2005). Before the V1309 Sco event, the only opportunity to test models of convective mergers had been to study putative merger products long after the mergers were over, by which time any debris had dissipated and any detectable evidence of circumstellar or merger-induced stellar activity had subsided.

\section{OBSERVATIONS}

\subsection{Pre-merger Observations}

A pre-merger IR detection was obtained by the Spitzer Space Telescope (Werner et al. 2004) IRAC camera (Fazio et al. 2004) as part of the GLIMPSE Galactic plane survey (Benjamin et al. 2003; Churchwell et al. 2009), in four bandpasses centered at 3.6, 4.5, 5.8, and $8.0 \mu \mathrm{m}$. We performed point-spread function (PSF)-fitting photometry on the GLIMPSE IRAC images with the Spitzer custom photometry software MOPEX's APEX script, while also fitting the nearby bright star and subtracting them with the apex-qa.nl script that produces a residual image.

The Spitzer IRAC images were taken on 2007 May 10. Nova Sco 2008 was discovered on 2008 September 2 (Nakano 2008), and peaked in visual brightness in early September of 2008. Data from the OGLE project (Udalski 2003) showed that the $I$ band brightness increase had begun in 2008 March (Tylenda et al. 2011). Thus the Spitzer images were obtained about 10 months before the first $I$-band brightness increase attributed to the merger, or about 16 months before peak brightness (which was $\sim 10$ mag in $V$ brighter than the pre-merger level).

\subsection{Post-merger Observations}

Following the merger, the remnant was observed in multiple scans each day by the WISE mission (Wright et al. 2010) on 
2010 March 19 and 20 in all four bandpasses, and several times in the three shorter-wavelength bandpasses during the period of 2010 September 15 through 20. The four WISE bandpasses are centered at about 3.6, 4.6, 12, and $22 \mu \mathrm{m}$, respectively. with a spatial resolution of $\sim 6$ arcsec pixel ${ }^{-1}$ in the three shortestwavelength bandpasses and $12 \operatorname{arcsec~pixel}^{-1}$ at $22 \mu \mathrm{m}$.

We obtained Spitzer images at several epochs in 2012 in the two short-wavelength channels (3.6 and $4.5 \mu \mathrm{m})$. The object was detected in both channels at all epochs. Images of V1309 Sco (three images in channels 1 and 3, two images in channels 2 and 4) were mosaicked together in each band with the Spitzer custom mosaicker and point-source extraction software package MOPEX mosaicker after performing overlap correction first. We then ran the point-source extraction module APEX of the MOPEX package to measure the flux.

We imaged V1309 Sco with the SIRIUS camera (Nagayama et al. 2003) on the IRSF $1.4 \mathrm{~m}$ telescope in Sutherland, South Africa, on several dates in 2011 and 2012. The first and the last sets were observed in $J, H$, and $K s$ bands simultaneously, while the second set used narrowband $\mathrm{Pa} \beta$ and $\mathrm{Br} \gamma$ filters, likewise simultaneously. Each of the three $\mathrm{HgCdTe}$ detectors of SIRIUS has a field of view of $7.7 \mathrm{arcmin} \times 7.7 \mathrm{arcmin}$ and pixel scale $0.45 \operatorname{arcsec}_{\text {pixel }}{ }^{-1}$. Observations were carried out in automatic dithering mode with $\sim 20$ arcsec steps. Individual frame exposure times were $30 \mathrm{~s}$, and total exposure times were typically $\sim 30$ to 60 minutes for the combined sets of exposures each night. All observations were taken in clear conditions, and with seeing of $\sim 1-1.2 \operatorname{arcsec}$ in the $K$ band. The data were reduced using conventional methods consisting of flat-fielding, sky construction and subtraction, and registration and co-adding of frames using dedicated IRAF and STARLINK packages.

We obtained near-IR images of V1309 Sco using the IRIS2 camera (Tinney et al. 2004) on the Anglo-Australian Telescope. Observations in the $J$ filter were made on 2011 March 23, and in the $J, H$, and $K$ filters on 2012 April 3-4, in seeing of 0.9 to 1.5 arcsec and non-photometric conditions. For each filter five $60 \mathrm{~s}$ images (dithered by 1 arcmin) were obtained, covering a $7.7 \times 7.7 \operatorname{arcmin}^{2}$ field of view at $0.45 \operatorname{arcsec}$ pixel $^{-1}$. Point sources were cross-referenced against the 2MASS Point Source Catalog to provide an astrometric and photometric calibration.

For the purpose of analysis using color-color plots, we converted measurements from $K$ to $K_{S}$ using the conversion for a red, low-mass object described by Rudy et al. (1996), who found that $K$ is about 0.11 mag brighter than $K_{s}$ for such objects. We assumed the case of a late-type star because the post-merger remnant in 2010 showed the spectrum of an M star (Mason et al. 2010).

We obtained imaging with the Hubble Space Telescope (HST) WFC3 on 2012 February 20 using the F160W filter, which effectively covers wavelengths from $1.4 \mu \mathrm{m}$ to $1.7 \mu \mathrm{m}$, and thus is similar to the Johnson $H$ band which covers $1.45-1.8 \mu \mathrm{m}$. The exposure time was $44 \mathrm{~s}$. We obtained a WFC3 image on 2012 February 19 using the F164N filter, which has a width of $0.209 \mu \mathrm{m}$ and includes the [Fe II] line at $1.64 \mu \mathrm{m}$, with an exposure time of $518 \mathrm{~s}$. We also obtained a WFC3 exposure of 2548 s on 2012 February 20 with the F673N narrow filter which covers a part of the Johnson $R$ bandpass including the [S II] lines at $6717 / 6731 \AA$. The [Fe II] and [S II] lines are commonly seen in shocked material near stellar outflows.

We obtained additional photometry in 2012 June, with the MORIS instrument at the NASA IRTF observatory. The MORIS bandpasses are very similar to Sloan $r$ and $i$. Seeing was $\sim 0.8$ arcsec. The signal-to-noise ratio $(\mathrm{S} / \mathrm{N})$ was over 100 in both bandpasses. We deconvolved the images of V1309 Sco and Star 5 using the IRAF PHOT task. We did not obtain photometry at the time of a photometric standard, so we calculated the magnitudes by using a comparison star which was not reported to vary in $R$ across the two (POSS) epochs of the USNO-B1.0 catalog. After converting from $i$ to $I$ and from $r$ to $R$, we obtained magnitudes of $I=17.5$ and $R=17.8$. USNO-B1.0 catalog photometric values may be substantially affected by a number of sources of error whose magnitude for any particular object is not known, and as discussed by Monet et al. (2003) are not, on the whole, highly reliable. Our comparison star's magnitude could be affected significantly either by catalog errors, or by possible intrinsic variations if it happens to be an unknown variable. We did not attempt to subtract possible flux contributions to the V1309 flux from any unresolved nearby stars (see Section 5.3).

We performed point-source extraction and photometry on the $H S T$, IRSF, and AAT data using the IRAF PHOT task and IDL's APER task. In the case of the IRSF and AAT images, the V1309 Sco PSF partially overlapped that of a nearby star. We fit the nearby star's PSF to a Gaussian and subtracted that from the images before performing photometric measurements of V1309 Sco. For each measurement, we used both PHOT and APER and used the average of the flux for the result. We found that the measured flux difference between the IRAF PHOT and IDL APER methods was less than $0.01 \mathrm{mag}$.

V1309 Sco was observed photometrically in $B, V, R, I, J, H$, and $K$ several times from 2008 October 13 until 2013 March by the SMARTS $1.5 \mathrm{~m}$ telescope (Walter et al. 2012). These data on V1309 Sco are available in the public SMARTS archive but have not been published until now. We show these data, together with our own $R, I$, and near-IR measurements, in Figure 1.

As a comparison star for ground-based IR photometric measurements, we used HD 316780, which has spectral type of about A6 (Ammons et al. 2006) and is about 2 arcmin from V1309 Sco. Its $J, H$, and $K_{s}$ magnitudes reported by 2MASS are $11.272 \pm 0.024,11.293 \pm 0.036$, and 11.282 \pm 0.03 , respectively, with no data quality warnings shown. It has not been reported in the literature or in any catalogs to be variable. Its DENIS magnitudes are $I=11.459 \pm 0.03, J=11.404 \pm$ 0.07 , and $K_{s}=11.277 \pm 0.08$. The DENIS catalog values exhibit a flux change of $\sim 0.1 \mathrm{mag}$ in $J$ at about the $1 \sigma-2 \sigma$ level over a timescale of a few years, but this is within our estimated uncertainty of $\sim 0.1 \mathrm{mag}$ in the PSF deconvolution. Also, the $I$ magnitudes reported by the DENIS survey and by the OGLE II program (Sumi et al. 2004) differ from one another by only $0.027 \mathrm{mag}$, which again represents only about a $1 \sigma$ difference in those catalog values. Thus the available archival data do not suggest that HD 316780 is variable enough in the near-infrared to significantly affect our photometric analysis.

\section{PRE-MERGER INFRARED FLUXES \\ 3.1. I-band Magnitudes}

The DENIS survey (Epchtein et al. 1994, 1997) covered the region of V1309 Sco in $I$ at some unspecified time in the late 1990s (specific observation dates are not given). The DENIS survey has nominal limiting magnitudes of $I=18.5$. The actual limiting magnitude could be a few magnitudes brighter than those values if photometric conditions were unusually poor, but conditions were good a large majority of the time during the survey (Paturel et al. 2003). Also, the effective limiting magnitude was affected if the field was crowded, as it is near the Galactic center (Unavane et al. 1998). The Galactic coordinates of V1309 Sco are $l=359.8 \mathrm{deg}, b=-3 \mathrm{deg}$. Unavane et al. 


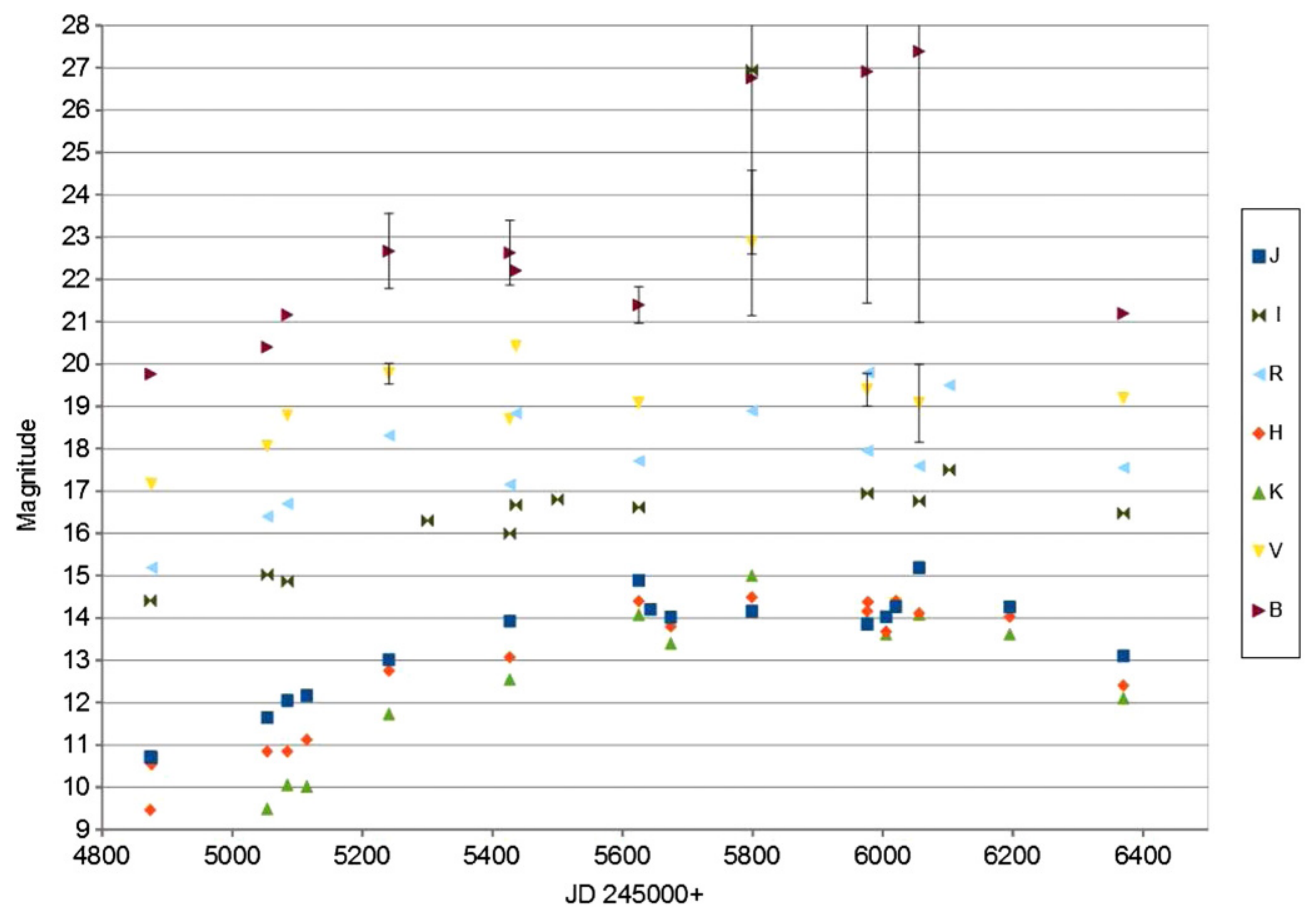

Figure 1. Magnitudes of V1309 Sco from 2009 February, after the 2008 optical outburst had subsided, until 2013 March. Error bars are smaller than the plot symbols except where shown. I-band measurements on days 5300, 5436, and 5500 are taken from Tylenda et al. (2011). Other data are from our own observations or from the SMARTS online data set. For comparison, the $I$-band magnitudes were $17>I>16$ during the 2001-2008 period before the main outburst (Tylenda et al. 2011).

(A color version of this figure is available in the online journal.)

(1998) found that in a field bounded by $l=0 \pm 5 \mathrm{deg}, b=0 \pm$ $1.5 \mathrm{deg}$ the survey had $80 \%$ completeness down to $I=17$.

V1309 Sco was monitored in the $I$ band by the OGLE project from August of 2001 until after the outburst (Tylenda et al. 2011). Tylenda et al. (2011) show that V1309 Sco exhibited complex variability in $I$ during the pre-outburst years of 2001 through 2007. During this time, its $I$ magnitude declined from $\sim 16.5$ at the beginning of OGLE monitoring to $\sim 17$ two years later, then gradually increased to $\sim 15.5$ before entering the outburst phase. The nominal DENIS limiting I magnitude would imply that the pre-merger system was $\sim 2$ mag fainter in the mid-1990s than in 2001. The DENIS crowded-field limiting magnitude, which is probably more representative of the V1309 Sco case, would imply that the pre-merger system was $\sim 0.5 \mathrm{mag}$ fainter in the 1990 s than in 2001. If one were to simply extrapolate the $I$ magnitude backward into the 1990s using the only first two years of OGLE monitoring, then one would conclude that V1309 Sco was $\sim 0.5$ mag brighter every two years earlier. However, a simple magnitude extrapolation from 2001 to the 1990s cannot be done with much confidence because of the complexity of the variations seen by OGLE during the pre-merger monitoring period.

It is possible that DENIS surveyed the field at a time when one of the pre-merger stars was being eclipsed. Tylenda at al. (2011) found that the period was approximately 1.4 days during the pre-outburst years, with an amplitude of $\sim 0.4$ mag during primary eclipses and $\sim 0.2$ mag during secondary eclipses, so if DENIS observed it during an eclipse then a nondetection might barely have occurred because of that.

Additional evidence that V1309 Sco was substantially fainter in $I$ prior to 2001 comes from Jaques \& Pimentel (2008), who found the progenitor object in the POSS-I E survey and measured an $I$ magnitude of 19 from a plate obtained in 1958 April.

\subsection{Limits on Near-infrared Magnitudes}

V1309 Sco was not detected by 2MASS (Skrutskie et al. 2006) observations which were obtained in 1998 August. The magnitude limits for inclusion in the 2MASS point-source catalog were $15.8,15.1$, and 14.2 for $J, H$, and $K_{s}$, respectively, with an $\mathrm{S} / \mathrm{N}$ of 10 or better required for inclusion. Also, it is not listed in the 2MASS Point Source Reject Table, for which the $\mathrm{S} / \mathrm{N}$ inclusion threshold was lowered to $\sim 3.5$. It is seen (Table 1) that during 2011 and 2012, the near-IR magnitudes of v1309 Sco remain $\sim 1-1.5$ mag brighter than the pre-merger upper limits inferred from the 2MASS nondetections.

It was also not detected by the DENIS survey, which had limiting magnitudes in $J$ and $K_{s}$ of 16.5 and 14.0, respectively.

\subsection{Spitzer Observations}

V1309 Sco is visible in GLIMPSE IRAC images obtained during the period of 2007 May 10-13, which was about a year before the visible-light outburst began. Because it is not listed in the GLIMPSE catalog, we performed photometry on the image using IRAF DAOPHOT. These 2007 flux densities are shown in Table 2.

Tylenda et al. (2011) concluded that the contact binary that merged included an early $\mathrm{K}$ giant, and post-merger optical spectra (Mason et al. 2010) showed that the remnant resembled an $\mathrm{M}$ giant. The spectral energy distributions (SEDs) of $\mathrm{K}$ and M stars peak in the $J$ to $K$ range and decline rapidly at longer wavelengths. In Table 3, we show the 2007 IRAC fluxes along with model stellar IR SEDs generated using the "Star-PET" stellar IR flux estimation tool provided by the Spitzer Science Center. The numbers in the table are normalized to the flux density of an M0 III star in the $3.6 \mu \mathrm{m}$ channel. The SED of V1309 Sco before the merger is $\sim 50 \%$ greater than expected for a late-type star having no circumstellar material in the $4.5 \mu \mathrm{m}$ 
Table 1

Near-infrared Broadband Magnitudes of V1309 Sco from Our Original Data

\begin{tabular}{|c|c|c|c|c|}
\hline Obs. Date & Post-peak Day Number ${ }^{a}$ & $J$ & $H$ & $K_{s}$ \\
\hline 1998 Aug $^{\mathrm{b}}$ & -3675 & 15.8 & 15.1 & 14.2 \\
\hline 2011 Mar 23 & 930 & $14.2 \pm 0.2$ & & \\
\hline 2011 Apr 23 & 961 & $14.02 \pm 0.1$ & $13.80 \pm 0.07$ & $13.40 \pm 0.05^{\circ}$ \\
\hline 2012 Feb 20 & 1264 & & $14.46 \pm 0.08^{\mathrm{d}}$ & \\
\hline 2012 Mar 19 & 1292 & $14.03 \pm 0.06$ & $13.68 \pm 0.06$ & $13.62 \pm 0.08^{\mathrm{c}}$ \\
\hline 2012 Apr 3 & 1307 & $14.27 \pm 0.08$ & $14.40 \pm 0.11$ & \\
\hline 2012 Apr 4 & 1308 & & & $14.35 \pm 0.13^{\circ}$ \\
\hline 2012 Sep 25 & 1483 & $14.26 \pm 0.05$ & $14.03 \pm 0.12$ & $13.62 \pm 0.06$ \\
\hline
\end{tabular}

Notes. Pre-merger upper limits from the 2MASS catalog are included for comparison. Magnitudes from the SMARTS catalog are not included in this table, but those data are available in the online SMARTS catalog, and are plotted in Figure 1.

${ }^{a}$ Number of days after the adopted date of peak nova visual brightness when observation was obtained. The exact date of peak brightness is not known. We have adopted 2008 September 5 for the date of the peak, which AAVSO and OGLE data imply is within $\pm \sim 5$ days from the date of the peak in visible wavelengths.

b 1998 values are pre-merger upper limits from the 2MASS catalog.

c Converted from Johnson $K$ magnitude.

d Converted from HST WFC3 F160W filter.

Table 2

Infrared Flux Densities of V1309 Sco at Different Epochs after the Merger

\begin{tabular}{|c|c|c|c|c|c|}
\hline Obs. Date ${ }^{\mathrm{a}}$ & Days Post-peak ${ }^{b}$ & $3.6^{\mathrm{c}} \mu \mathrm{m}$ & $4.5 \mu \mathrm{m}$ & $12 \mu \mathrm{m}$ & $22 \mu \mathrm{m}$ \\
\hline 2007 May 10 & ( $\sim 1$ yr pre-merger) & $6.0 \pm 0.2$ & $4.3 \pm 0.15$ & $4.2 \pm 0.22^{\mathrm{d}}$ & \\
\hline 2010 Mar 15 & 556 & $124.0 \pm 3.0$ & $673 \pm 13$ & $2562 \pm 25$ & $2920 \pm 30$ \\
\hline 2010 Sep 15 & 740 & $63.0 \pm 6.0$ & $453 \pm 44$ & $2350 \pm 35$ & \\
\hline 2012 May 19 & 1352 & $4.3 \pm 0.5$ & $83.0 \pm 0.5$ & & \\
\hline 2012 Jun 20 & 1383 & $4.2 \pm 0.4$ & $80.0 \pm 2.0$ & & \\
\hline 2012 Oct 28 & 1513 & $2.0 \pm 0.4$ & $68.0 \pm 1.0$ & & \\
\hline 2012 Dec 2 & 1548 & $2.2 \pm 0.2$ & $66.0 \pm 1.0$ & & \\
\hline
\end{tabular}

Notes. Flux densities are in mJy. 2007 flux densities in the $12 \mu \mathrm{m}$ and $22 \mu \mathrm{m}$ columns are from IRAC channels 3 and 4 which do not exactly match the two long-wavelength WISE bandpasses.

a 2010 flux densities are from the WISE catalog. 2007 and 2012 flux densities are from Spitzer measurements.

b Day number defined in the same way as in Table 1.

c The IRAC and WISE bandpasses have steep declines in the sensitivity near each of the bandpass cutoffs. The IRAC effective sensitivity, to the nearest $0.1 \mu \mathrm{m}$, is from $3.2 \mu \mathrm{m}$ to $3.9 \mu \mathrm{m}$ in channel 1 and from $4.0 \mu \mathrm{m}$ to $5.0 \mu \mathrm{m}$ in channel 2. The two shortest WISE bandpasses are centered at $3.4 \mu \mathrm{m}$ and $4.6 \mu \mathrm{m}$. According to the WISE documentation those bandpasses were designed to have "close correspondence to" the two shortest IRAC channels, with the $3.4 \mu \mathrm{m}$ bandpass being effectively "slightly" bluer and the $4.6 \mu \mathrm{m}$ bandpass "slightly" redder.

d IRAC channel 4 has an effective bandpass of approximately $6.5 \mu \mathrm{m}$ to $9 \mu \mathrm{m}$. The flux density from IRAC channel 4 is included in this table as the closest IRAC bandpass to the WISE band 3 that is centered at $12 \mu \mathrm{m}$ and covers approximately from $7.5 \mu \mathrm{m}$ to $17 \mu \mathrm{m}$.

Table 3

Relative Model Stellar IR Flux Densities of Unreddened Stars in the Four IRAC and Two MIPS Instrument Bandpasses, Compared to Pre-merger IRAC Flux Densities of V1309 Sco

\begin{tabular}{lcccccc}
\hline \hline Spectral Type & $3.6 \mu \mathrm{m}$ & $4.5 \mu \mathrm{m}$ & $5.8 \mu \mathrm{m}$ & $8 \mu \mathrm{m}$ & $15 \mu \mathrm{m}$ & $24 \mu \mathrm{m}$ \\
\hline V1309 Sco & 1.0 & 0.72 & 0.79 & 0.70 & & \\
M0 III & 1.0 & 0.48 & 0.37 & 0.24 & 0.07 & 0.02 \\
KV III & 0.98 & 0.48 & 0.37 & 0.22 & 0.07 & 0.01 \\
K0 III & 0.98 & 0.52 & 0.37 & 0.22 & 0.07 & 0.04 \\
\hline
\end{tabular}

channel, and almost $3 \times$ greater than that of a late-type star in the $8 \mu \mathrm{m}$ channel. The IRAC SED shows a significant peak in the $5.8 \mu \mathrm{m}$ channel. It is not known whether this peak represents a broad peak from thermal emission.

Messineo et al. (2012) constructed an extensive set of infrared color-color plots of a diverse set of evolved stars, including red supergiants and different types of AGB stars, which they showed could be used to compare and classify stars by their IR colors.
In the [3.6]-[8.0] versus [3.6]-[4.5] color-color diagram of Messineo et al. (2012), evolved stars as a group show redder [3.6] - [8.0] colors for a given value of [3.6]-[4.5]. In the diagram of Messineo et al. (2012), V1309 Sco is located in the region containing $\mathrm{SiO}$ stars and red supergiants, but apart from the region of $\mathrm{OH} / \mathrm{IR}$ stars. Messineo et al. (2012) interpret increasingly red colors in their plot as probably resulting from a combination of increasing mass loss rates and of increasing water absorption around $3.6 \mu \mathrm{m}$, as well as the effects of other features such as $\mathrm{SiO}$ absorption near $4 \mu \mathrm{m}$, and $\mathrm{CO}_{2}$ absorption near $4.3 \mu \mathrm{m}$ (Cami 2002) that tend to move a star blueward in this color-color diagram.

Ybarra \& Lada (2009) showed that the IRAC [3.6]-[4.5] versus [4.5]-[5.8] plane can be used to identify the presence of intermediate-excitation shocked $\mathrm{H}_{2}$ gas in stellar outflows by its unique location that is separated from stellar values in the color-color plane. They plotted a region in this color-color plane bounded by $1500 \mathrm{~K} \leqslant T \leqslant 4000 \mathrm{~K}$ and $n_{\mathrm{H}}$ from $10^{3}$ to $10^{5} \mathrm{~cm}^{-3}$. The pre-merger IRAC colors of V1309 Sco are found in their 
plot to be well separated from the region occupied by shocked $\mathrm{H}_{2}$ and thus is not consistent with the presence of that emission line. We also compared the IRAC colors to those of a set of models of protostellar jets from low-mass stars calculated by Takami et al. (2010, 2012). The pre-merger [3.6]-[4.5] versus [3.6]-[5.8] colors are not consistent with either thermal $\mathrm{H}_{2}$ emission or shock-induced $\mathrm{H}_{2}$ emission according to those models.

In the IRAC [3.6]-[8.0] versus [8.0] color-magnitude diagram of Srinivasan et al. (2009), the pre-merger colors of V1309 Sco place it among the reddest stars in the region populated by O-rich and C-rich AGB stars.

Optical spectra obtained during the outburst showed vigorous mass loss including significant amounts of heavy elements which could lead to the production of dust and complex molecules such as are seen in AGB stars. For example, Rudy et al. (2008a, 2008b) and Mason et al. (2010) detected the presence of $\mathrm{H}_{2} \mathrm{O}, \mathrm{CO}$, and heavy elements in absorption. IRAC colors are not in themselves sufficient to argue that the $\mathrm{K}$ giant companion was in an AGB phase, although that cannot be ruled out in the absence of spectra obtained before the merger. However, these colors do imply that enough circumstellar material was present well before the outburst to at least mimic the IRAC colors of evolved objects having thick, chemically enriched circumstellar shells. This is consistent with the prediction by dynamical models of substantial mass loss in the orbital plane prior to a merger (e.g., D'Souza et al. 2006).

\section{POST-MERGER OPTICAL AND INFRARED BRIGHTNESS VARIATIONS}

Optical and near-IR magnitudes of V1309 Sco from the period 2009 February 11 until 2013 March 17 are shown in Figure 1. With few exceptions, discussed later, the SED increases in flux toward longer wavelengths (including the mid-IR) at all epochs.

The light curve of the months-long nova outburst phase has already been studied and discussed by other authors (e.g., Mason et al. 2010; Tylenda et al. 2011). The purpose of this section is to consider the long-term behavior of the remnant after the outburst.

No pre-merger near-IR detections of V1309 Sco are available. It is seen in Figure 1 that the near-IR magnitudes after the nova remain $\sim 2$ mag brighter than the pre-merger upper limits placed by nondetections in the 2MASS and DENIS surveys.

Infrared flux densities are shown in Table 2.

Nicholls et al. (2013) published WISE data of V1309 Sco obtained in 2010 March, but did not consider the WISE data of V1309 Sco which were obtained in 2010 September. From the pre-merger nondetections by the IRAS and AKARI surveys, Nicholls et al. (2013) found that the WISE flux densities represented at least a six-fold increase from pre-merger values at those wavelengths. By comparing with the 2007 IRAC flux densities, we find (Table 3) that in 2010 March V1309 Sco had increased in brightness by $\sim 20 \times$ in the $3.6 \mu \mathrm{m}$ region, by $\sim 156 \times$ in the $4.5 \mu \mathrm{m}$ region, and by $\sim 600 \times$ in the $8-12 \mu \mathrm{m}$ region compared to 2007 levels. Nicholls et al. (2013) attempted to fit the 2010 May WISE and ground-based mid-IR photometry to a simple dust model, but with what they regarded as poor results. Nevertheless, they concluded that the mid-IR SED was consistent with that of dusty environment in 2010 that probably included large grains.

Several time-dependent trends of interest are seen in Figure 1. The brightness at all magnitudes continues to decline rather steadily in all bandpasses from 2009 February until 2010
February 13, while the color indices remain roughly the same. Starting with the next set of measurements, obtained on 2010 August 18, the optical and near-IR light curves are decoupled. The BVRI brightness stops declining and even increases by roughly a magnitude, while the $J H K$ brightness continues to decline by about two magnitudes from 2010 February to 2011 March 4. The brightness in the $J H K$ region next enters a relatively constant period from about 2011 March until 2012 May. Yet during about the same period, the three sets of optical measurements starting with the measurement on 2011 August 25 through 2012 May 8 show a brightness decrease in $B$ of about $5 \mathrm{mag}$, from 2011 March 4 to 2011 August 25, with a decline of about three magnitudes in $V$ at a single epoch. Finally, it is seen that the $J H K$ brightness shows a steady increase of about 2 mag from 2012 May 8 to 2013 March 17, again apparently decoupled from the brightness changes in the optical bandpasses. The optical brightness during the time of near-IR brightness increase remains at roughly similar levels to those seen in 2009 and 2010 before the "big dip."

Although the various BVRI color indices remain roughly constant outside of the "big dip," they exhibit significant variability from epoch to epoch. For example, the value of $R-I$ has a mean of $1.26 \pm 0.46$ even when the extremely faint values of 2010 are ignored. Because $R$ and $I$ are the optical bandpasses least affected by extinction, this variability suggests that either fluxes in these bandpasses are strongly affected by emission lines, or the extinction is highly variable.

Another noteworthy event is a single epoch in which $J>$ $H>K$, followed by one set of measurements in which the $K$ flux is brighter than the $H$ flux (no $J$ measurement at that epoch was obtained). This occurs at the epoch where the $B$ brightness has suddenly declined. At other epochs, $K>H>J$. It is also evident that during the "big dip" period, the magnitude differences between $J, H$, and $K$ are significantly smaller than they had been before, with the $J$ to $K$ range showing a gradual increase toward pre-"dip" values during the two sample epochs after the "big dip."

Since early 2012, the $J, H$, and $K$ brightness has shown an increasing trend, while the $3.6 \mu \mathrm{m}$ and $4.5 \mu \mathrm{m}$ fluxes have steadily decreased. Further, the ratio of the $4.5 \mu \mathrm{m}$ to $3.6 \mu \mathrm{m}$ flux density monotonically increased from about 4 to about 34 from 2010 May to 2012.

The dramatic drop in $B$ brightness in 2011 to 2012, which is echoed at only one epoch in $V$ and is generally not exhibited in the other bandpasses, might be caused by reddening from a relatively sudden appearance of dust in the line of sight. Such dust might either come from dust formation or from a dusty mass crossing the line of sight. Because the nova outburst would be expected to propel material generally outward and disrupt any orbiting material already present, we think that this photometric event more likely results from dust formation.

We suggest that the most likely explanation for the apparent decoupling of the near-IR photometric behavior from the optical photometric behavior is that the near-IR emission is so dominated by emission lines that any underlying contribution from a dust continuum remains relatively insignificant even when there is a dust formation event. A strong contribution from variable emission lines would also explain the unusual behavior of the relative magnitudes of $J, H$, and $K$ during the period when the $B$ magnitude faded by several magnitudes. If a dust formation event were precipitated by a shock, one might expect some of the common near-IR shock lines (e.g., Hartigan et al. 2004) to be in strong emission at those epochs. 
Also, it is somewhat surprising that the IR fluxes at $3.6 \mu \mathrm{m}$ and $4.5 \mu \mathrm{m}$ were much higher in 2012 than before the merger at the same time that the $I$ magnitude had declined to values similar to the pre-merger magnitudes, because one might expect that the observed strong post-merger IR excess would come from material that would be dense enough to increase the extinction shortward of $\sim 1 \mu \mathrm{m}$. Also, it is not expected that an SED representing only continuum emission should rise by a factor of $\sim 34$ from the $3.6 \mu \mathrm{m}$ bandpass to the $4.5 \mu \mathrm{m}$ bandpass, as those channels are virtually contiguous. A reasonable hypothesis is that these channels are being strongly affected by emission lines. One possible emission source in those bandpasses could be the IR shock lines from molecular H (Noriega-Crespo et al. 2004).

A long-term trend of diminishing brightness is seen from 2010 through 2012. The time sampling is sparse enough that we do not rule out the possibility of some temporary brightness increases having occurred during those years. The decrease is much larger in the $3.5 \mu \mathrm{m}$ bandpass, which has declined by a factor of about 62 since 2010, than in the 4.5/4.6 $\mu \mathrm{m}$ bandpass, which has declined by a factor of about 10 . This, too, could be naturally explained if these bandpasses were strongly affected by emission lines.

\subsection{Near-infrared Colors}

The remnant had the optical spectrum of an $\mathrm{M}$ giant about one year after the merger (Mason et al. 2010). The remnant had been evolving in apparent spectral type during the year after the merger. Its spectral type since 2010 has not been observed so it is uncertain whether the remnant still has an apparent spectral type of M. Near-IR observations of V1309 Sco since 2008 show that the location of the remnant in the $J-H$ versus $H-K_{s}$ plane has remained much redder than the location of the $\mathrm{K}$ or $\mathrm{M}$ giants which do not have circumstellar material (e.g., Lee 1970; Chen et al. 1988; Messineo et al. 2012). Evolved stars can appear redder in this plane either from circumstellar dust or because of continuum absorption by water in the $H$ band (Groenewegen $\&$ de Jong 1993; Messineo et al. 2012).

The $Q 1$ parameter is a quantitative measure of the deviation from the reddening vector in the $H-K_{s}$ versus $J-K_{s}$ plane caused by circumstellar material, independent of interstellar reddening (Messineo et al. 2005 and references therein). Messineo et al. (2012) showed that this parameter is sensitive enough to distinguish between different types of evolved stars having substantial mass loss. We calculated the values of the $Q 1$ parameter for all of the epochs for which $J, H$, and $K$ are known. For a typical evolved, mass-losing star, the value of the parameter is not expected to change substantially except over stellar evolutionary timescales. However, the values of $Q 1$ for V1309 Sco vary greatly without a clear pattern over time, ranging from -1.64 to +1.03 . For $\mathrm{K}$ and $\mathrm{M}$ giants without mass loss, $Q 1$ typically ranges from 0.41 to 0.68 , and from 0.44 to -1.56 for stars with strong mass loss such as AGB stars, red supergiants, and OH/IR stars (Messineo et al. 2012). The rapid and extreme changes of the $Q 1$ parameter of V1309 Sco since the merger could be explained if the near-IR bandpasses used were being affected by strong, variable emission lines.

\subsection{WISE Colors}

The WISE magnitudes of V1309 Sco, as observed in 2010 March, are $[3.5]=8.49,[4.6]=6.02,[12]=2.73$, and [22] $=$ 1.15 , with magnitude uncertainties of $\sim 1 \%$ in each bandpass.
No detailed study of the locations of AGB stars in the WISE color-color planes, and the possible use of those colors for the classification of different types of AGB stars, has not yet been discussed in publication. However, Wright et al. (2010) showed that "normal" main sequence stars have WISE colors of [3.4]-[4.6] $\leqslant \sim 0.7$ and [4.6]-[12] $\leqslant \sim 1.5$. V1309 Sco is much redder in its WISE colors at both epochs in 2010 than main sequence stars would be. Its WISE colors are also much redder than those of a sample of S stars (C.-W. Tsai 2013, private communication), which are late-type giants thought to be in an intermediate stage between red giants and AGB stars. The postmerger value of $W 1-W 2=2.485$, which is greatly different from the pre-merger color difference of $W 1-W 2 \sim 0.1$. The models of Takami et al. $(2010,2012)$ show that this large value of $W 1-W 2$ could be due to polycyclic aromatic hydrocarbon emission, thermal $\mathrm{H}_{2}$ emission, or shock lines.

\section{WFC3 IMAGES AND FLUXES}

\subsection{Constraints on Possible Extended Emission}

The spectra of Mason et al. (2010) showed variable outflows with typical speeds of $\sim 300-500 \mathrm{~km} \mathrm{~s}^{-1}$, and as fast as $\sim 2000-3500 \mathrm{~km} \mathrm{~s}^{-1}$ in $\mathrm{H} \alpha$ at one epoch. Those velocities

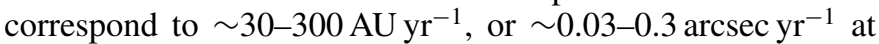
the distance of V1309 Sco (Tylenda et al. 2011). WFC3 images have pixel scales of 0.13 arcsec in the IR channels and 0.04 arcsec in the UVIS channel. If a substantial amount of material had been ejected in 2008 and had continued to move outward since then at velocities of a few thousand $\mathrm{km} \mathrm{s}^{-1}$, and were bright enough, it could be resolvable by the time our WFC3 images were obtained.

Assuming $E(B-V)=0.55$ as estimated by Mason et al. (2010), assuming that the flux is the same across the bandpass, and binning over a single pixel, our exposures place the following upper limits on extended emission. In the broad F160W bandpass (approximately Johnson $H$ ), our exposure would detect a flux of $15 \mu \mathrm{Jy} \operatorname{arcsec}^{-2}$ with an $\mathrm{S} / \mathrm{N}$ of 5 . In the narrow F164N ([Fe II] $1.64 \mu \mathrm{m}$ ) bandpass, we find an upper limit of $200 \mu \mathrm{Jy} \operatorname{arcsec}^{-2}$ for $\mathrm{S} / \mathrm{N}=5$. In the narrow F673N bandpass (part of the Johnson $R$ band), we find an upper limit $2.5 \times 10^{-19} \mathrm{erg} \mathrm{cm}^{-2} \mathrm{~s}^{-1} \operatorname{arcsec}^{-2}$ for $\mathrm{S} / \mathrm{N}=5$.

The images of the V1309 region are shown in Figures 2, 3, and 4. We do not find a significant departure from a pointsource PSF, so we conclude that no extended material was detected. This places an upper limit of radius of $\sim 120 \mathrm{AU}$ on any extended material emitting in the F673N filter, and $~ 400 \mathrm{AU}$ in the $H$ band. Without detailed modeling, we cannot place a quantitative limit on the mass of extended material which these spatial limits imply.

\subsection{WFC3 Magnitudes}

In the F160W filter we measured an $\mathrm{AB}$ magnitude of $15.83 \pm 0.08$ using aperture photometry. The uncertainty stated here is the formal uncertainty from Poisson noise in the image. This corresponds to a Johnson $H$ magnitude of $14.46 \pm 0.08$. Some error in the conversion to an $H$ magnitude could result from emission or absorption in the part of the Johnson $H$ filter not covered by the F160W filter, because the F160W filter covers approximately 1.40 to $1.70 \mu \mathrm{m}$, while the $H$ filter covers approximately 1.30 to $1.70 \mu \mathrm{m}$.

We measured the F673N flux magnitude to be $\mathrm{AB}=20.36 \pm$ 0.07 . The F673N filter is centered at $6764.5 \AA$. It is effectively $\sim 14 \AA$ wide, and was designed to measure the [S II] doublet at 


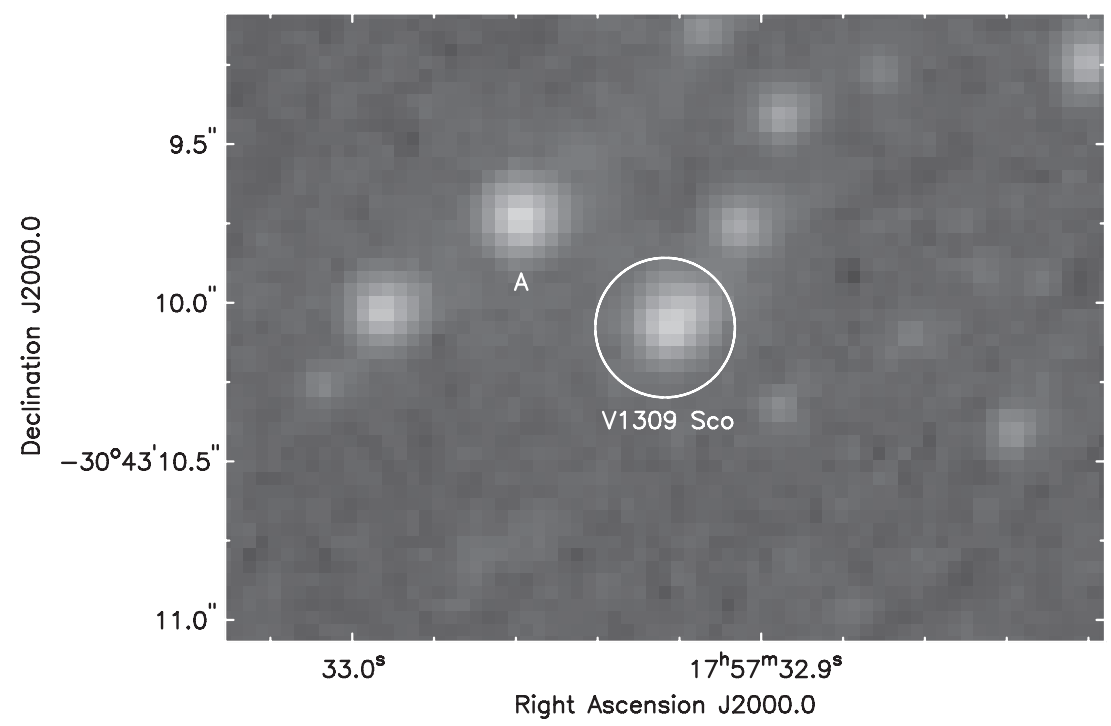

Figure 2. WFC3 F673N image of V1309 Sco region. V1309 Sco is circled in white. The flux is displayed in log scale. Object A is discussed in the text.

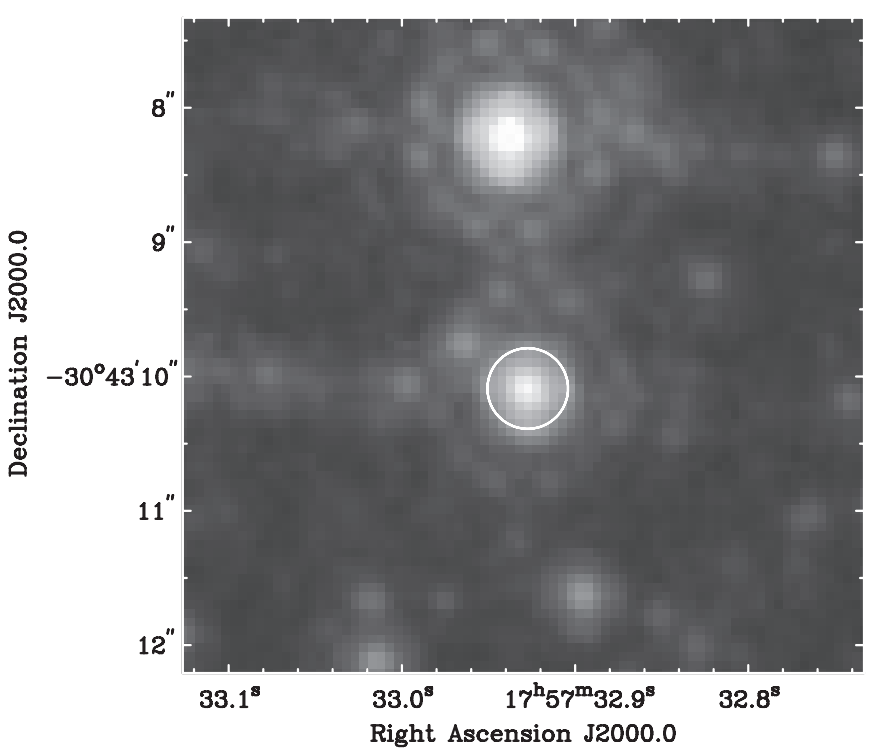

Figure 3. WFC3 F164N image of V1309 Sco and its immediate area. V1309 Sco is circled in white. The flux is in log scale.

$6717 / 6731 \AA$. It thus measures a $14 \AA$ section of the Johnson $R$ band. V1309 Sco is spatially well resolved in this filter, and has an $\mathrm{S} / \mathrm{N}$ of $\sim 90$. Although the flux of V1309 Sco in this filter is measured with high precision, in the absence of spectra it is uncertain how accurately the flux measurement in this filter represents the continuum value or the $R$ magnitude. A significant uncertainty results from the question of how much, if any, [S II] emission is present. Because our near-IR spectra show a number of shock lines, it is possible that there is also shock-induced [S II] emission. The interpretation of the flux is further complicated by the fact that $\mathrm{M}$ stars typically have a strong $\mathrm{TiO}$ absorption feature which can cover much of the F673N bandpass. An inspection of several M star spectra in the literature shows that this absorption feature could suppress the flux measurement in the F673N filter by up to $\sim 50 \%$. It is possible that both $\mathrm{TiO}$ absorption and $[\mathrm{S}$ II] emission are present in V1309 Sco.

We did not calculate a broadband magnitude from the F164N filter because that is a narrow filter $(17.48 \mathrm{~nm})$ centered on the

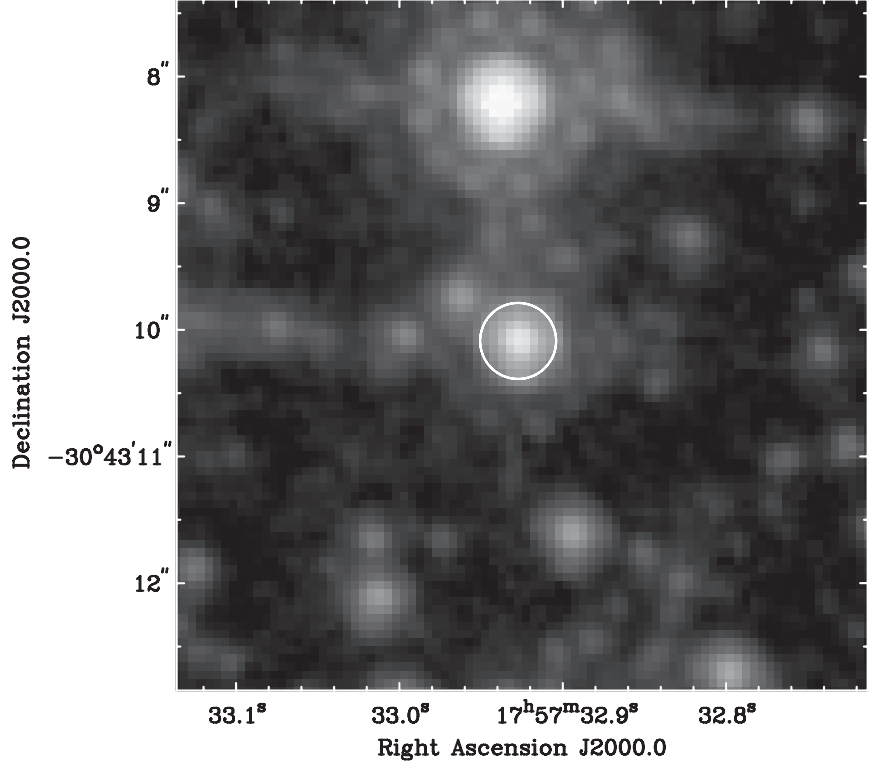

Figure 4. F160W (WFC3 $H$ band) image of the V1309 Sco region. V1309 Sco is circled in white. The flux is in log scale.

[Fe II] emission line at $1645 \mathrm{~nm}$. Fe II and [Fe II] emission lines are commonly seen wherever there is a low-density medium heated by shocks or by expanding atmospheres (e.g., Baschek 1998). Fe II is the dominant ionic state over most of the cooling region of a typical shock (Hartigan et al. 2004), and [Fe II] emission at $1645 \mathrm{~nm}$ is typically one of the strongest near-IR emission lines from a shocked region, so strong [ $\mathrm{Fe}$ II] emission might be expected in the merger remnant environment. As discussed elsewhere in this paper, there is reason to suspect that strong emission lines such as [Fe II] were present in V1309 Sco at the time of the WFC3 observations.

\subsection{Discovery of an Uncataloged Star Close to V1309 Sco}

We measure the J2000 position of V1309 Sco in the F160W image to be $17^{\mathrm{h}} 57^{\mathrm{m}} 32^{\mathrm{s}} .930,-30^{\circ} 43^{\mathrm{m}} 10^{\mathrm{s}} 09$. This is matched exactly by one of the objects in the F673N image. WFC 3 coordinates are stated to have an astrometric uncertainty of \pm 0.05 arcsec. The coordinates given by Nakano (2008), 
measured when the nova was near peak visible brightness, are $17^{\mathrm{h}} 57^{\mathrm{m}} 32^{\mathrm{s}} .93 \pm 0.01,-30^{\circ} 43^{\mathrm{m}} 10^{\mathrm{s}} .1 \pm 0.10$.

The WFC3 F673N image reveals an uncataloged object about 0.5 arcsec northeast of V1309 Sco. This is labeled "Star A" in Figure 2. Its flux in this filter is a few percent greater than that of V1309 Sco. Its $\mathrm{J} 2000$ coordinates are $17^{\mathrm{h}} 57^{\mathrm{m}} 32^{\mathrm{s}} .96$, $-30^{\circ} 43^{\mathrm{m}} 09^{\mathrm{s}} .73$. Its distance from V1309 Sco is small enough that it could contaminate photometric and spectroscopic measurements that do not have sufficient spatial resolution. Its PSF is consistent with a point source. We measure its magnitude in the F160W filter to be $\mathrm{AB}=18.7 \pm 0.3$, corresponding to a Johnson $H$ magnitude of 17.35 . We mention this object because it represents a source of possible contamination when groundbased photometry of V1309 Sco is performed.

The F673N image also shows a fainter but well resolved object approximately 0.25 arcsec northwest of V1309 Sco. Examination of the F160W image using a histogram equalization flux display scale shows another object, which is barely detected and barely resolved in this filter. We do not estimate its infrared magnitude because it is detected at an $\mathrm{S} / \mathrm{N}$ of $\sim 2$ in the F160W image. It is somewhat brighter relative in the F673N image than in the F160W image, which suggests that it is probably not an M star.

A source of uncertainty in estimating the possible flux contribution of these objects is their unknown nature. Many objects of similarly faint $R$ magnitudes in the direction of the Galactic center may be expected to be red dwarfs, which can vary by a few tenths of a magnitude because of stellar activity, or distant red giants, which can vary periodically by up to a few magnitudes in brightness if they are highly evolved stars such as Miras.

An upper limit to the amount of variability introduced by these objects, in a situation where they are not resolved in ground-based photometry and affect the measured brightness of V1309 Sco, might be inferred from the scatter in the $I$ magnitudes observed in V1309 Sco in the OGLE observations before the merger. According to the OGLE project online documentation, seeing at the OGLE site is typically around 0 .'7. No mention is made by Tylenda et al. (2011) regarding possible contamination by other stars which could not easily be resolved, so it is not clear whether the OGLE photometric measurements include contributions from any of the nearby stars discussed here. A visual inspection of the light curve of Tylenda et al. (2011) shows that the "random" scatter has a maximum amplitude of $\sim 0.5 \mathrm{mag}$ in $I$. This approximate limit to the variable contribution from unresolved sources does not, of course, imply that there is not a larger systematic error in the I magnitudes reported by OGLE.

\section{SUMMARY AND DISCUSSION}

Optical, near-infrared, and mid-infrared photometry of V1309 Sco reveals a complicated photometric evolution occurring over more than a decade.

Its $I$-band brightness was at least $\sim 0.5$ mag less in 1998 than in 2001, and $\sim 2.5$ mag less in 1958 than in 2001. The few existing N-body models of mergers of contact binaries show disks of ejected material forming and increasing in mass over timescales of weeks before the merger, but not timescales of decades. If the suggestion by Tylenda et al. (2011) is correct that the generally increasing $I$-band brightness observed during several years from 2002 until Nova Sco 2008 was caused by ejected material, then models of pre-merger mass loss will require more refinement. It is possible that mechanisms other than mass loss might produce an increasing I-band brightness, for example, perhaps the heating of part of the stellar photospheres. Spitzer IRAC observations in 2007, before the merger, show an SED presenting a large flux excess in the 3-8 $\mu$ range over what would be expected of a $\mathrm{K}-\mathrm{M}$ star having no circumstellar material. Also, the location of V1309 Sco matches that of highly evolved stars with large amounts of chemically complex mass loss, such as AGB stars, when the 2007 Spitzer colors and the near-IR colors are evaluated with standard color-color diagnostics.

After the nova, its I-band brightness generally declined in optical and near-IR bandpasses until 2010, when the optical brightness increased by about one magnitude over several months while the $J, H$, and $K$ brightness continued to decline by about a magnitude. Sometime in 2011 between March and September, the brightness dropped by about $4 \mathrm{mag}$ in $B$ and $V$, while the relative flux ratio of $J$ and $K$ reversed, with $K$ becoming about 1.5 mag fainter. Since early 2012, the optical magnitudes have remained approximately the same as before the event of 2011, during which time the near-IR magnitudes have increased by about 2 mag and the flux densities in the $3.6 \mu$ and $4.5 \mu \mathrm{m}$ Spitzer channels have declined slightly.

There are several peculiarities of the time behavior and the SED of V1309 Sco since the merger. First, the longterm $J, H$, and $K$ magnitude changes appear to be mostly decoupled from the $B V R I$ magnitude changes. Second, the $J-H$ and $H-K$ colors, and the value of the $Q 1$ parameter which measures circumstellar reddening, vary greatly and irregularly over timescales of a few months or less. Third, the ratio of the $4.5 \mu \mathrm{m}$ flux density to the $3.6 \mu$ flux density is unreasonably large for a pure continuum contribution to two such narrow contiguous bandpasses, especially in 2012. Fourth, during 2011 and 2012 the brightness in the $J H K$ region has steadily increased while the flux densities in the $3.6 \mu \mathrm{m}$ and $4.5 \mu \mathrm{m}$ filters have steadily decreased. All of these unusual aspects of the IR flux behavior could readily be explained if the bandpasses were dominated by some of the many emission lines, including shock lines commonly seen in high-speed stellar outlfows, that are found in the range of 1-5 $\mu \mathrm{m}$ (e.g., Hartigan et al. 2004). It would be reasonable to expect that material ejected outward in the nova would collide with circumstellar material formed before the merger, so that shocks could originate. We have recently obtained near-IR spectra of V1309 Sco which show such emission lines (B. McCollum et al., in preparation). The photometric behavior of V1309 Sco since 2008 suggests that these lines may have been strongly in emission, and that the emission has been variable, since a few months after the merger.

V1309 Sco shows evidence of having a large amount of chemically enriched, circumstellar material after the merger which is continuing to evolve in ways that are not understood. To our knowledge, there is as yet no modeling in the literature that predicts spectra or SEDs of merger remnants, or that quantitatively predicts the photometric and spectroscopic evolution of a merger before, during, and after the event. V1309 Sco offers a valuable opportunity to compile a data set that will be an essential foundation to a new generation of merger models. We are continuing to monitor V1309 Sco photometrically, and have begun to obtain optical and infrared spectra in order to learn more about the nature, circumstellar environment, and long-term evolution of this rare object. An ultimate goal is to discover photometric or spectroscopic signatures that could be used to identify other stellar mergers in the future during or soon after the merger, and perhaps to identify with more certainty older merger remnants. 
This work is based on observations made with the NASA/ ESA Hubble Space Telescope, which is operated by the Association of Universities for Research in Astronomy, Inc., under NASA contract NAS 5-26555. These observations are associated with program 12684. Support for program 12684 was provided by NASA through a grant from the Space Telescope Science Institute, which is operated by the Association of Universities for Research in Astronomy, Inc., under NASA contract NAS 5-26555. This research has made use of the SIMBAD database and the VizieR catalog access tool, CDS, Strasbourg, France, and the NASA/IPAC Infrared Science Archive, which is operated by the Jet Propulsion Laboratory, California Institute of Technology, under contract with the National Aeronautics and Space Administration. This work is based on observations obtained with the Spitzer Space Telescope, which is operated by the Jet Propulsion Laboratory, California Institute of Technology, under a contract with NASA. This publication makes use of data products from the Wide-field Infrared Survey Explorer, which is a joint project of the University of California, Los Angeles, and the Jet Propulsion Laboratory/California Institute of Technology, funded by the National Aeronautics and Space Administration. This publication makes use of data products from the Two Micron All Sky Survey, which is a joint project of the University of Massachusetts and the Infrared Processing and Analysis Center/California Institute of Technology, funded by the National Aeronautics and Space Administration and the National Science Foundation. The DENIS project was supported in France by the Institut National des Sciences de l'Universe, the Education Ministry, and the Centre National de la Recherche Scientifique, in Germany by the State of Baden-Wrtemberg, in Spain by the DGICYT, in Italy by the Consiglio Nazionale delle Ricerche, in Austria by the Fonds zur Förderung der Wissenschaftlichen Forschung and the Bundesministerium für Wissenschaft und Forschung. We thank Peter Barnes for making available some of the AAT observing time for this project. We thank Tim Brooke for a useful discussion on performing photometry in IRAC images, Chao-Wei Tsai for giving us access to his unpublished work on WISE colors of stars, and David Gilbank for obtaining some of the observations.

Facilities: WISE, HST (WFC3), IRSF, Spitzer, AAT

\section{REFERENCES}

Ammons, S. M., Robinson, S. E., Strader, J., et al. 2006, ApJ, 638, 1004

Baschek, B. 1988, in IAU Colloq. 94, Physics of Formation of Fe II Lines Outside LTE, ed. R. Viotti, A. Vittone, \& M. Friedjung (Dordrecht: Riedel), 1

Benjamin, R. A., Churchwell, E., Babler, B. L., et al. 2003, PASP, 115, 953

Buchholz, R. M., Schodel, R., \& Eckart, A. 2009, A\&A, 499, 483
Cami, J. 2002, PhD thesis, Univ. Amsterdam

Chen, P. S., Gao, H., Chen, Y. K., \& Dong, H. W. 1988, A\&AS, 72, 239

Churchwell, E., Babler, B. L., Meade, M. R., Whitney, B. A., et al. 2009, PASP, 121,213

Dale, J. E., Davies, M. B., Church, R. P., \& Freitag, M. 2009, MNRAS, 393, 1016

D’Souza, M. C. R., Motl, P. M., Tohline, J. E., \& Frank, J. 2006, ApJ, 643, 381 Epchtein, N., de Batz, B., Capoani, L., et al. 1997, Msngr, 87, 27

Epchtein, N., de Batz, B., Copet, E., et al. 1994, Ap\&SS, 217, 3

Fazio, G. G., Hora, J. L., Allen, L. E., et al. 2004, ApJS, 154, 10

Freitag, M., \& Benz, W. 2005, MNRAS, 358, 1133

Genzel, R., Thatte, N., Krabbe, A., et al. 1996, ApJ, 472, 153

Groenewegen, M. A. T., \& de Jong, T. 1993, A\&A, 267, 410

Hartigan, P., Raymond, J., \& Pierson, R. 2004, ApJ, 614, 69

Jaques, C., \& Pimentel, E. 2008, IAUC, 8972

Lee, T. A. 1970, ApJ, 162, 217

Leonard, P. J. T. 1989, AJ, 98, 217

Lohr, M. E., Norton, A. J., Kold, U. C., et al. 2012, A\&A, 542, A124

Martin, E. L., Spruit, H. C., \& Tata, R. 2011, A\&A, 535, 50

Mason, E., Diaz, M., Williams, R. E., Preston, G., \& Bensby, T. 2010, A\&A, 516, 108

Mateo, M., Harris, H. C., Nemec, J., \& Olszewski, E. W. 1990, AJ, 100, 469

Messineo, M., Habing, H. J., Menten, K. M., et al. 2005, A\&A, 435, 575

Messineo, M., Menten, K. M., Churchwell, E., \& Habing, H. 2012, A\&A, 537, A10

Monet, R., Levine, S. E., Canzian, B., et al. 2003, AJ, 125, 984

Nagayama, T., Nagashima, C., Nakajima, Y., et al. 2003, Proc. SPIE, 4841, 459 Nakano, S. 2008, IAUC, 8972

Nicholls, C. P., Melis, C., Soszyński, I., et al. 2013, MNRAS, 431, L33

Noriega-Crespo, A., Morris, P., Marleau, F. R., Carey, S., et al. 2004, ApJS, 154,352

Paturel, G., Petit, C., Rousseau, J., \& Vauglin, I. 2003, A\&A, 405, 1

Pols, O. 2009, in Stellar Mergers Workshop, Leiden, Netherlands, http://www.lorentzcenter.nl/lc/web/2009/350/presentations/Pols.pdf

Rasio, F. A. 1995, ApJL, 444, L41

Robertson, J. A., \& Eggleton, P. P. 1977, MNRAS, 179, 359

Rudy, R. J., Lynch, D. K., Russell, R. W., et al. 2008a, IAUC, 8976

Rudy, R. J., Lynch, D. K., Russell, R. W., et al. 2008b, IAUC, 8997

Rudy, R. J., Rossano, G. S., \& Puetter, R. C. 1996, ApJL, 458, L41

Skrutskie, M. F., Cutri, R. M., Stiening, R., et al. 2006, AJ, 131, 1163

Srinivasan, S., Meixner, M., Leitherer, C., et al. 2009, AJ, 137, 4810

Stepien, K. 2011, A\&A, 531, 18

Sumi, T., Wu, X., Udalski, A., et al. 2004, MNRAS, 348, 1439

Takami, M., Chen, H.-H., Karrk, J. L., et al. 2012, ApJ, 748, 8

Takami, M., Karr, J. L., Koh, H., Chen, H.-H., \& Lee, H.-T. 2010, ApJ, 720,155

Tinney, C. G., Ryder, S. D., Ellis, S. C., et al. 2004, Proc. SPIE, 5492, 998

Tutukov, A. V., \& Yungelson, L. R. 1987, Ap\&SS, 130, 15

Tylenda, R., Hajduk, M., Kaminski, T., et al. 2011, A\&A, 528, 114

Udalski, A. 2003, AcA, 53, 291

Unavane, M., Gilmore, G., Epchtein, N., et al. 1998, MNRAS, 295, 119

Walter, F. M., Battisti, A., Towers, S. E., Bond, H. E., \& Stringfellow, G. S. 2012, PASP, 124, 1057

Webbink, R. F. 1976, ApJ, 209, 829

Webbink, R. F. 1985, in Interacting Binary Stars, ed. J. Pringle \& R. Wade (Cambridge: Cambridge Univ. Press), 39

Werner, M. W., Roellig, T. L., Low, F. J., et al. 2004, ApJS, 154, 1

Wright, E. L., Eisenhardt, P. R. M., Mainzer, A. K., et al. 2010, AJ, 140, 1868

Ybarra, J. E., \& Lada, E. A. 2009, ApJL, 695, L120 